\title{
3D Optical Body Scanning: Application to Forensic Medicine and to Maxillofacial Reconstruction
}

\author{
Gianluca CAVAGNINI, Giovanna SANSONI*, Angelo VERTUAN, and Franco DOCCHIO \\ Laboratory of Optoelectronics, University of Brescia, Brescia, Italy
}

\begin{abstract}
This paper reports the results of the experience carried out at our Laboratory in the fields of forensic medicine and of maxillofacial reconstruction. Optical 3D digitizers have been used in the tests. The point clouds have been edited and modeled following suitable reverse engineering and rapid prototyping methodologies. The aim in the forensic application was to assess the feasibility of these techniques in view of the in-situ documentation of crime scenes. In particular, it was considered of interest to acquire and model the scene before its removal, and to measure the victim body during post mortem examination. The aim in maxillofacial reconstruction was to develop an efficient process for fabricating the prosthetic element, avoiding patient stress and optimizing both the aesthetical appearance and the functionality of the prosthesis.
\end{abstract}

Keywords: forensic medicine, prosthetic reconstruction, maxillofacial defects, 3D optical measurement, reverse engineering, rapid prototyping

\section{Introduction}

The aim of this paper is to present the main results of the research carried out at our Laboratory in the fields of forensic medicine and of maxillofacial reconstruction, using optical 3D range sensors, reverse engineering and rapid prototyping techniques. The former activity began upon request of the Italian State Police in 2004. The goal was to assess the feasibility of using non contact sensors (i) for documenting crime scenes before their alteration, and (ii) for measuring lesions on cadavers in post mortem analysis.

The latter activity began upon request of maxillofacial specialists, in 2007. The aim was to develop the procedure for producing facial prosthetic elements to reduce patient's discomfort and dependence on the anaplasthologist skill, and to increase the process efficiency and performance.

Over the years, we have carried out a noticeable number of experiences in both the researches. On one hand, we have been helped by the experience previously accumulated at the Laboratory in the frame of 3D optical acquisition, reverse engineering and metrological characterization, especially in applications typical of mechanical, manufacturing and automotive industry [1,2]. On the other hand, we were excited by the opportunity of exploiting our experience even in fields far from the industrial production, such as those related to crime scenes and to maxillofacial reconstruction.

Although the methods used to perform the study are well known in production fields, their experimentation in the applications considered here is recent. As far as autopsy examination is concerned, the literature has pointed out the use of radio-diagnostic tests, such as scanning electron microscopy (SEM), magnetic resonance imaging (MRI) and computer tomography systems (CT), to improve the quality of detail on the shape and size of tools in bone lesions [4]. In the last years, the analysis of bite marks has been approached by using photogrammetry methods [5, 6]. The use of pure reflective 3D optical sensors has only recently been published in forensics, for example to verify the consistency of wound morphology with the tool used [7, 8].

The interest in investigating their potential has grown over the years, mainly because they (i) show higher speed of analysis with respect to 3D SEM and CT, (ii) are available on the market at by far lower costs with respect to CT or MRI systems, (iii) present very good measurement performances in the macroscopic range, and (iv) are designed to perform the measurement in harsh conditions. They are rugged and portable and can be used on-site (i.e., directly on the crime scene, to analyze the victim in short times).

In maxillofacial prosthetic reconstruction, CT and MRI systems have been proposed for the prosthetic restoration of facial defects $[9,10]$. They represent a significant evolution with respect to traditional techniques [11], since any contact with the patient's face is avoided, and a very detailed description of tissues and shapes is achieved. However the are very invasive and expensive.

Only recently the use of 3D optical sensors have been proposed to safely acquire patient's face segments, in view of modeling and prototyping prosthetic elements [12-14].

* giovanna.sansoni@ing.unibs.it; +39- 0303715446; www.optolab-bs.it 
This trend is justified by (i) their non-invasiveness for the patient, due to the pure-reflective approach to the measurement, (ii) their acquisition speed, which increases the patient comfort and guarantees the accuracy of the measurements despite the unavoidable patient movements, (iii) their market availability at by far lower costs with respect to CT/MRI systems, and (iv) their performances in terms of data quality, system portability and ruggedness.

This paper is composed as follows. Section 2 describes the method followed to perform the experiments. Section 3 describes novel results of the experience performed during post mortem examination. To have a complete overview of our work, the interested reader will find more information in publications [16, 17]. Section 4 presents the results of prosthetic reconstruction of an ear. In this experience, the process has been optimized with respect to the work performed until 2009 [18, 19].

\section{Method}

\subsection{The acquisition device}

In the experiments we used the Vivid 910 digitizer (Konica Minolta, Inc) shown in Fig.1. The principle of measurement is based on laser triangulation [3]. The object is scanned by a plane of laser light coming from the Vivid's source aperture. The plane of light is swept across the field of view by a mirror, rotated by a precise galvanometer. Each scan line is observed by a single frame, captured by the CCD camera. The contour of the surface is derived from the shape of the image of each reflected scan line. The entire area is captured in 8 seconds ( 0.3 seconds in FAST mode). A brilliant (24-bit) color image is captured at the same time by the same CCD.
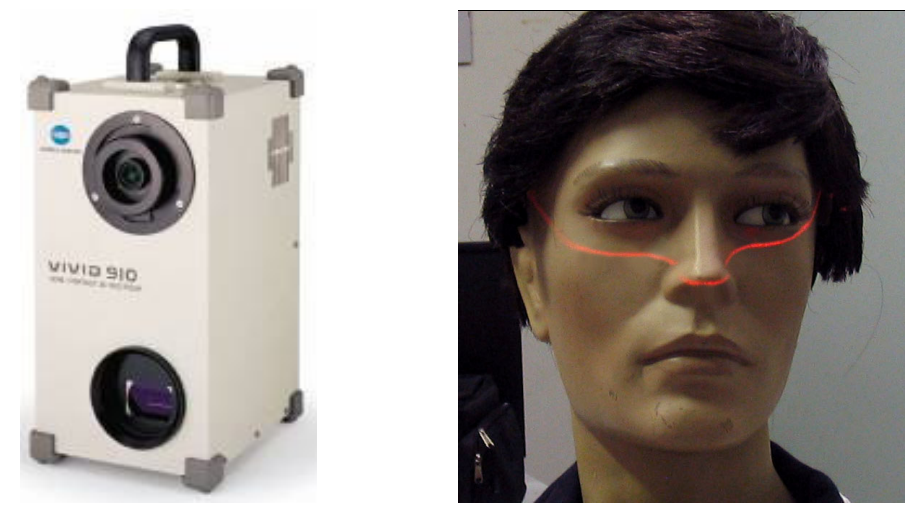

Fig. 1: The optical digitizer Minolta Vivid 910 (left); example of stripe deformation (right).

The system is rugged, portable, and extremely compact. It can be mounted on a tripod and properly oriented into the measurement volume to optimize the acquisition view-point. It is equipped with three lenses at focal lengths of $8 \mathrm{~mm}$ (Wide configuration), $14 \mathrm{~mm}$ (Middle configuration) and $25 \mathrm{~mm}$ (tele configuration) respectively. One of the main advantages of the instrument is that it does not require a phase of calibration: this feature is particularly relevant in the presented study cases, in which adaptability and capability of rapidly varying the measurement setup is often crucial. Typical values of the measurement parameters are listed in Table 1.

Table 1. Typical values of the measurement parameters of the Vivid910 device. FOV: Field of View, WD: Working Distance, Zrange: Range of measurement, Rz: Measurement resolution.

\begin{tabular}{lllll}
\hline Focal lengths & FOV $\left(\mathrm{mm}^{2}\right)$ & WD $(\mathrm{mm})$ & Zrange $(\mathrm{mm})$ & $\mathrm{Rz}(\mathrm{mm})$ \\
\hline Wide & $1000 \times 1000$ & 2000 & 1000 & 0,65 \\
Middle & $600 \times 450$ & 1000 & 500 & 0,3 \\
Tele & $140 \times 100$ & 600 & 200 & 0,13 \\
\hline
\end{tabular}

\subsection{The elaboration process of 3D data}

The process of measurement and of data elaboration consists of the following tasks:

1. The acquisition of the point clouds. To digitize complex scenarios it is necessary to perform multiple acquisitions from different point of views and with different degrees of detail in order to completely acquire the different areas.

2. The multi-view registration. It consists in the alignment of the set of the captured point clouds into a common reference frame. The module ImAlign, from the suite of software tools of Polyworks, (InnovMetric, Ca) accomplishes this task (http://www.innovmetric.com). 
3. The creation of the triangle-mesh. The 3D data of the point cloud are merged into a model that includes information about the topological contiguity of the points. This phase is performed by the use of ImMerge module of Polyworks.

4. The editing of the polygon model. The model is elaborated to (i) decrease the noise influence, (ii) measure point-to-point distances, sections and areas, (iii) render the 3D representation for further VMRL interaction, and (iv) topologically control the model for prototyping applications. This phase is performed by the use of ImEdit module of Polyworks.

5. The production of physical copies of the surfaces by means of rapid prototyping techniques.

\section{The experience in forensic medicine}

In 2005, the Italian Ministry funded the project entitled "Crime scene: application of optical measurement methodologies for the accurate, contactless acquisition, for the three-dimensional modelling and for the analysis". In this project, we worked together with forensic scientists, at the LABANOF Laboratory of the Institute of Legal Medicine of the University of Milan, to provide a full study of body lesions during the post-mortem investigation.

The main objective was to study the measurement performance of optical acquisition during the autopsy. Autopsy rooms are surprisingly crowded rooms, where multiple autopsies are carried out in parallel, forensic scientists and technicians work together, following precise protocols, and corpses arrive into and leave the room with a noticeable (at least for us!) frequency. Hence, the time available for the measurement is quite limited. In addition, the environment is highly illuminated, the autopsy table is very reflective, and corpses present an extremely high variation in colour and texture; hence measurement conditions are not favorable.

From our point of view, we expected to develop an efficient methodology for the elaboration of acquired raw data, in view of their fruitful analysis and manipulation by operators with minimum computer science training, and to provide significant study cases to the specialists in order to have their feed-back about both the quality of the measurements, and the effective usefulness of information extracted from our 3D data, with respect to the typology of information and documentation that is currently used for legal purposes.

From the point of view of legal doctors, the main objective was to understand the characteristics of 3D optical acquisition, its main strengths and weaknesses, in view of using it in real crime scenes, i.e., before the removal of the victim body. In fact, as they explained us, autopsies take place several days after the crime. Despite the corpse is kept at low temperature, tissues undergo modifications in their morfology: this is very critical, especially in the presence of induced lesions, such as those from weapons, knifes or blunt instruments. Thus, it was thought to be extremely interesting to have the possibility of examining the victim before these modification occurred.

\subsection{The cases of study}

Three cases are described in the following. The first concernes the measurement of a corpse in good state; the second one concerns the acquisition of a decomposed corpse. The third case of study is the measurement of a lesion.

\subsubsection{Case of study 1}

The objectives of this test were (i) to assess the measurement sensitivity in the presence of small scratches on the right hand of the corpse, and (ii) to verify the sensitivity of the measurement to steep variations in shape and color.

The region of the scratches is shown in Fig. 2. The digitizer was configured in the TELE mode; it was at about $1 \mathrm{~m}$ from the surface. The point cloud was acquired in 8 seconds. It was then elaborated to produce the mesh shown in Fig. 2.b. 


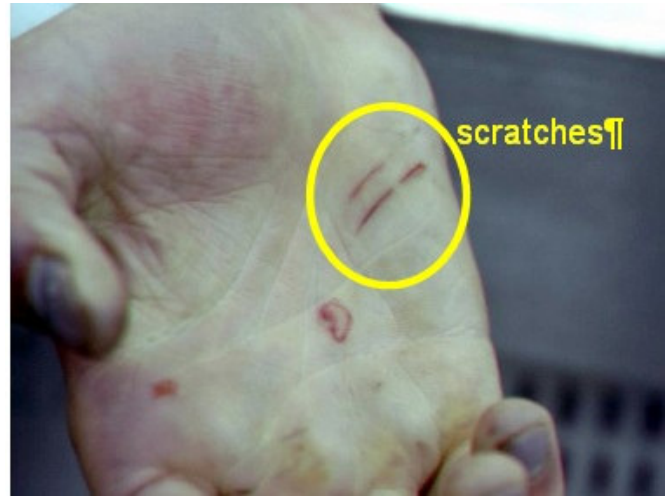

(a)

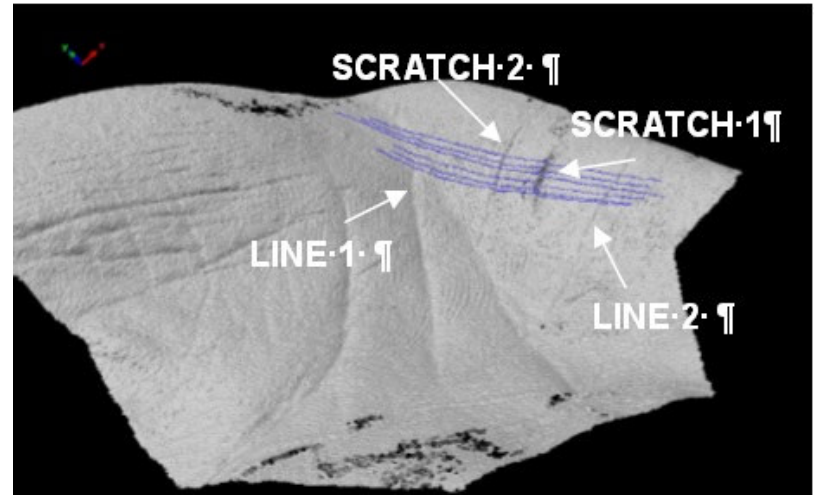

(b)

Fig. 2: Scratch analysis. (a) Image of the right hand. (b): corresponding mesh.

We were really surprised by the adherence of the model to the original shapes: in fact, we did not expect to observe even the lines of the hand in the mesh. To quantitatively study this model, we drew a number of sections (the blue lines in Fig. 2.b), including both the lines of the hand and the scratches. Fig. 3 presents the corresponding profiles.

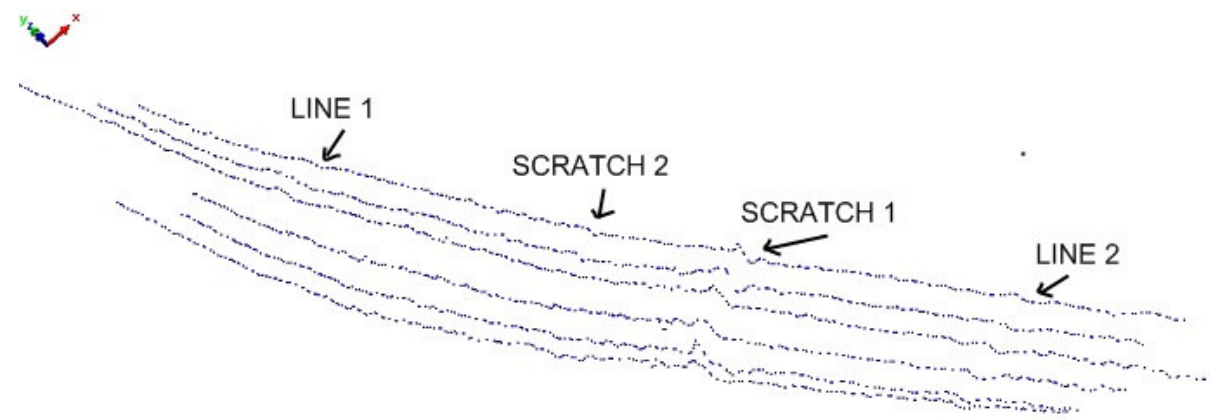

Fig. 3: Profiles of the sections extracted from the mesh in Fig. 2.b.

LINE 1 and LINE 2 appear as smooth concavities along each section. Their depth is lower than $0,5 \mathrm{~mm}$. In contrast, SCRATCH 1 is characterized by a peak followed by a small depression. The distance between them is $0,8 \mathrm{~mm}$. SCRATCH 2 is very thin. The measurement performed reveals that its height is about the same as for normal hand lines; however it can be distingushed from them by looking at its shape, which has a higher frequency content.

To verify the robustness of the acquisition to steep variations in shape and color, we performed the measurement in correspondence with the thorax region shown in Fig. 4. Since a large amount of water was present in the tissues, the surface is highly reflective. It was of concern to understand the ability of the laser source to comply with this situation. In this case, the Vivid 910 was set in the WIDE configuration, to acquire all the FOV in a single view. The mesh resulting from this acquisition is shown in Fig. 5.a (rendered shape) and Fig. 5.b (shape plus color).

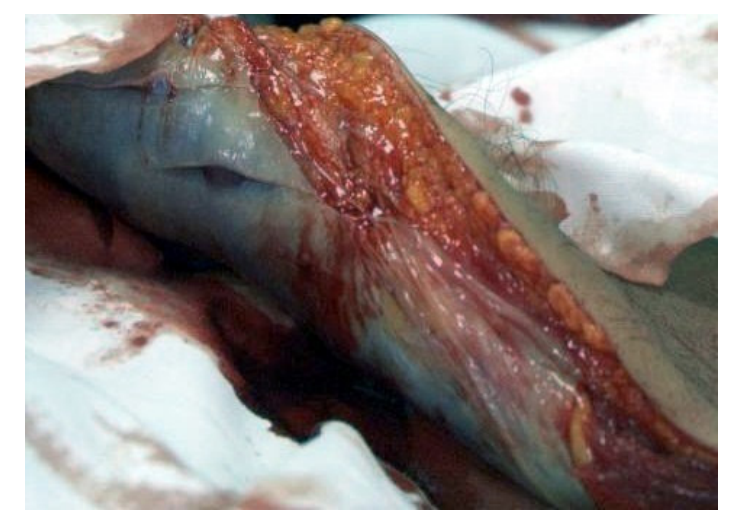

Fig. 4: The thorax region of the corpse during the autopsy. 


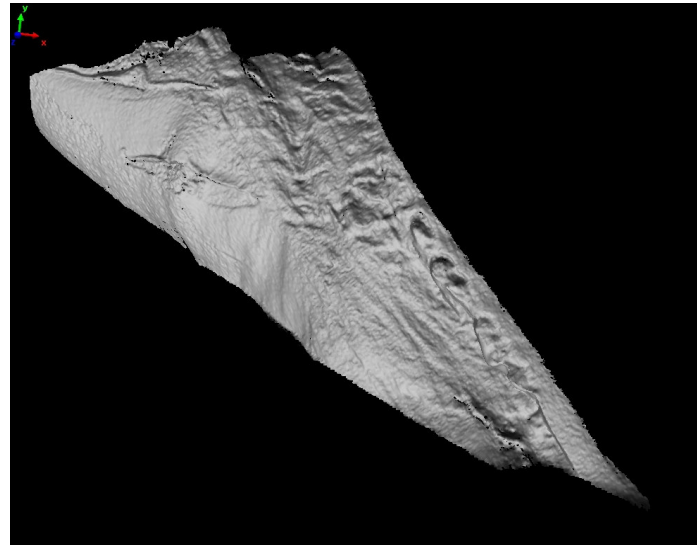

(a)

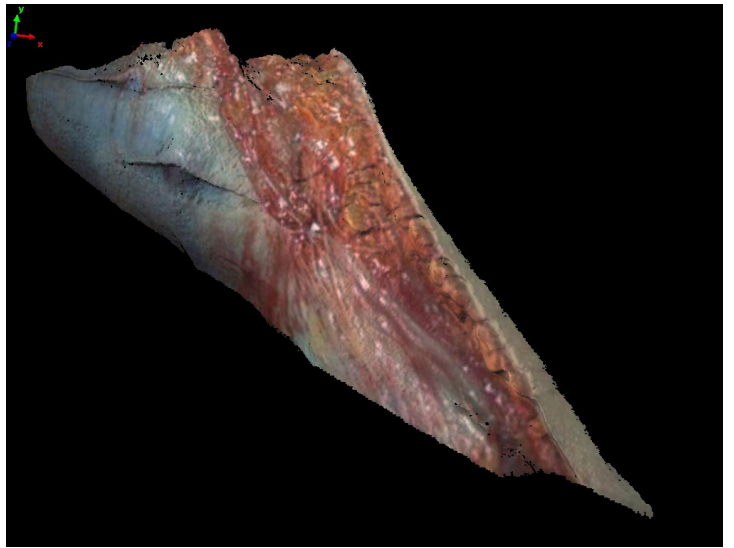

(b)

Fig. 5: Mesh corresponding to the surface in Fig. 4. (a): rendered view; (b) shape plus color.

The quality of the mesh is impressive; it has been disturbed by neither the reflectivity of the tissues, nor the variations of the shapes.

\subsubsection{Case of study 2}

During the tests we had the opportunity to acquire the corpse shown in Fig. 6. In this case, the body showed large variations in color which, due to decomposition, goes from black, to violet, red, and green. We set up the Vivid 910 in the MIDDLE configuration, and acquired the surface in a single view. The area interested by the measurement is framed in the figure. The result of the elaboration is shown in Fig. 7.

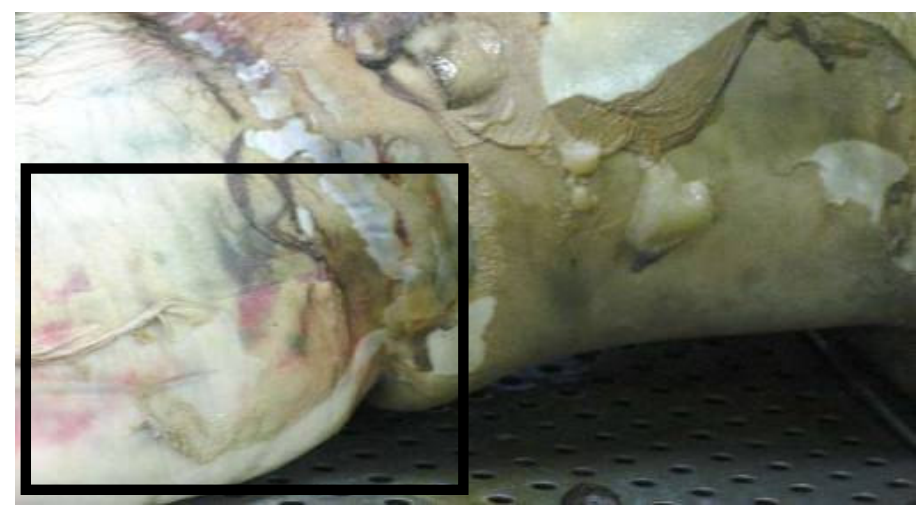

Fig. 6. Body segment of the decomposed corpse.

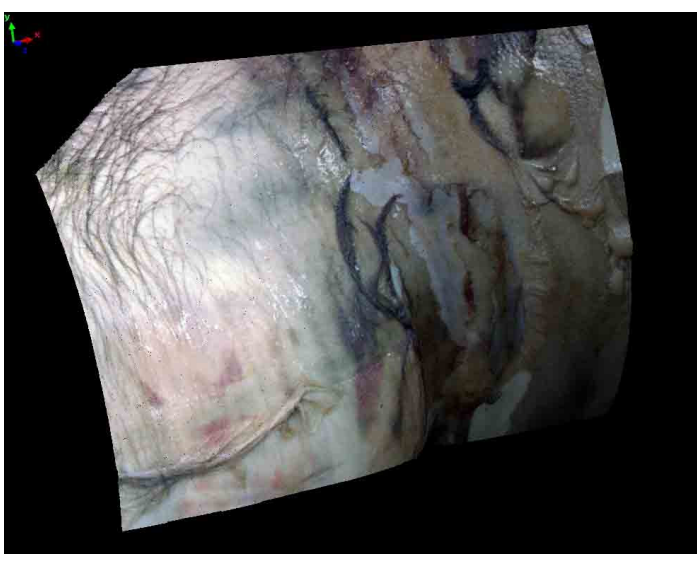

(a)

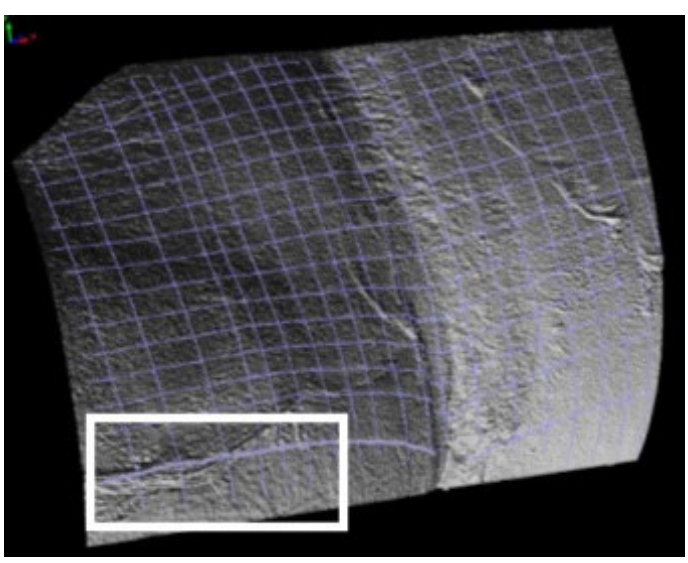

(b)

Fig. 7. Mesh corresponding to the surface in Fig. 6. (a): rendered view; (b): shape plus color. 
Both the color and the 3D shape are retained in the mesh. To better appreciate the resolution of the measurement, we drew the sections visible in Fig. 7.b. Fig. 8 presents the detail of the section framed in Fig. 7.b. The metric grid overlapped has a step of $1 \mathrm{~mm}$. The resolution of the section is $0,5 \mathrm{~mm}$.

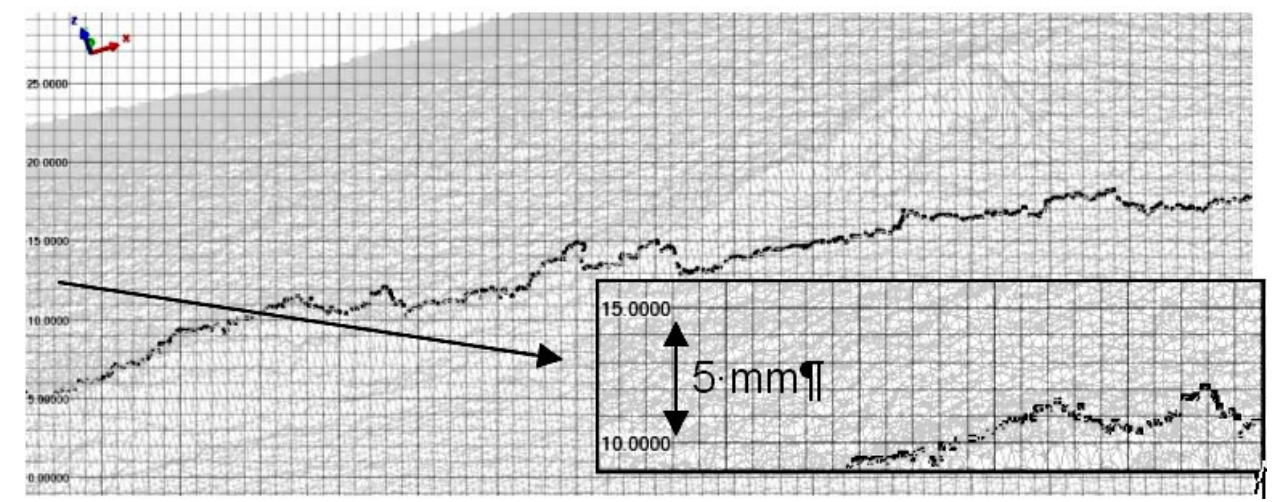

Fig. 8. Graph of the section framed in Fig. 7b. The resolution of the measurement is $0,5 \mathrm{~mm}$.

\subsubsection{Case of study 3}

The aim if this experience was to capture the lesion in Fig. 9, and to study the characteristics of the resulting mesh. This was considered extremely interesting by forensic scientists, in view of subsequent analysis. In fact, an objective, quantitative representation of the lesion would allow them to measure its dimension and shape more precisely than what they can do in normal conditions, i.e., by using contact rulers with millimeter resolution. In addition, the possibility of acquiring the shapes without touching them could make the difference when the problem is to guess the tool used by the offender.

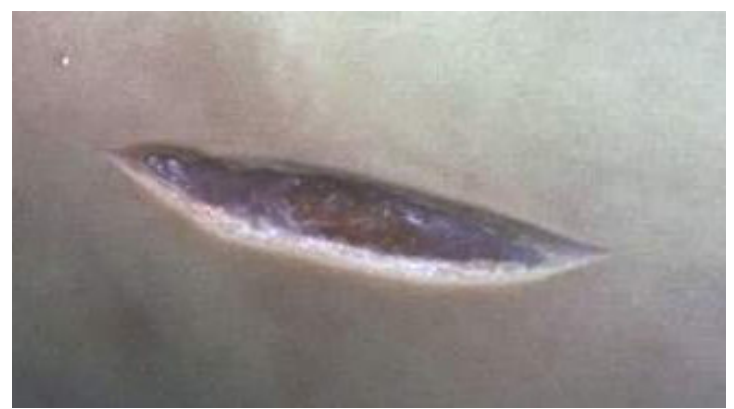

(a)

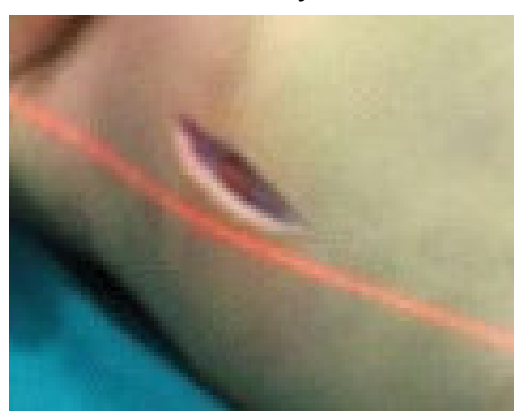

(b)

Fig. 9. Case of study 3. (a): Image of the lesion. Its length is $54 \mathrm{~mm}$. (b): Laser stripe from the Vivid 910 device.

In this case of study, we had the opportunity of gauging the lesion by using, besides the Vivid 910 device, even a different 3D optical digitizer. The system is shown in Fig. 10.a. It is a prototype developed at the Laboratory, called OPL-3D [20]. It is based on the projection of incoherent fringe patterns. A projector projects a sequence of eleven fringe patterns of incoherent light onto the object, following the well-known Gray Code-Phase Shift method. The video camera captures the patterns. These ones appear deformed by the object shape, as shown in Fig. 10.b.

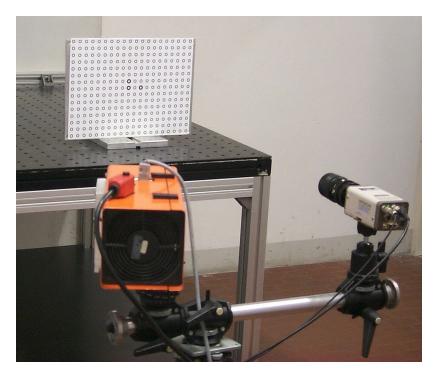

(a)

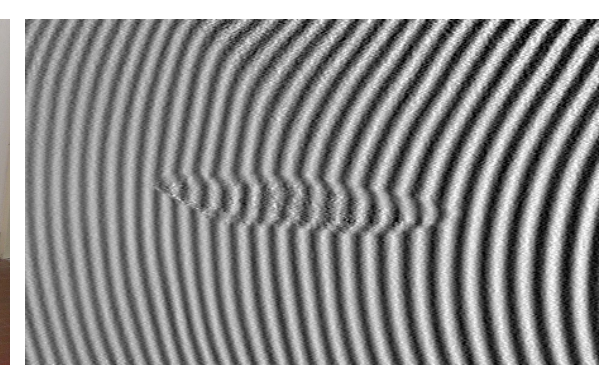

(b)

Fig. 10. The optical digitizer OPL-3D. (a): image of the device; (b): example of pattern deformation. 
Suitable developed procedures extract the shape of the object from the pattern deformation, in the form of point clouds, retaining either point coordinates and intensity or RGB color information. Dense point clouds are produced in $2 \mathrm{~s}$ for the acquisition, plus $4 \mathrm{~s}$ for the elaboration (data storage included). Table 2 reports typical values of three measurement configurations.

Table 2. Typical values of the measurement parameters of OPL-3D. FOV: Field of View, Zrange: Range of measurement, Rz: Measurement resolution.

\begin{tabular}{llll}
\hline Set up & FOV $\left(\mathrm{mm}^{2}\right)$ & Zrange $(\mathrm{mm})$ & $\mathrm{Rz}(\mathrm{mm})$ \\
\hline Set-up1 & $450 \times 350$ & 150 & 0,3 \\
Set-up2 & $300 \times 230$ & 100 & 0,2 \\
Set-up3 & $160 \times 123$ & 40 & 0,1 \\
\hline
\end{tabular}

The two devices show a number of similarities, and, at the same time, they present significant differences. In fact, both devices are based on active triangulation; they are designed to perform measurements in 'close range' (from few centimeters to one meter), and show resolution performances that match those required to gauge medium-small details as those typical of wounds and lesions. However, they differ in the light source. The former uses coherent light, characterized by high visibility of the stripe, and robustness against environmental illumination and surface color variations. The latter uses incoherent light, that results in reduced robustness against ambient illumination and surface color. On the other hand, the Vivid 910 system produces speckles, which could represent a limit in relation to the measurement precision. In contrast, using OPL-3D, speckles are absent.

The Vivid 910 is a 'close' system, in the sense that it is not possible to modify its optical geometry to adapt to the measurement problem; in contrast, OPL-3D is very flexible: the triangulation parameters (baseline and camera-projector orientation) can be finely adjusted in view of optimizing the measurement performance. However, this can be achieved by means of suitable system calibration, which in turn requires specific operator skill.

Hence, our interest was to compare the meshes from the two devices, and make experience with both systems, to appreciate their pros and cons, in relation to the application.

The mesh obtained from the Vivid 910 is shown in Fig. 11.a. It was acquired in a single view, with the Vivid 910 in the TELE configuration, at $60 \mathrm{~cm}$ from the surface. The mesh from OPL-3D is presented in Fig. 11.b. Again, a single view was sufficient to capture the surface. The device was configured in the set-up 3 of Table 2.

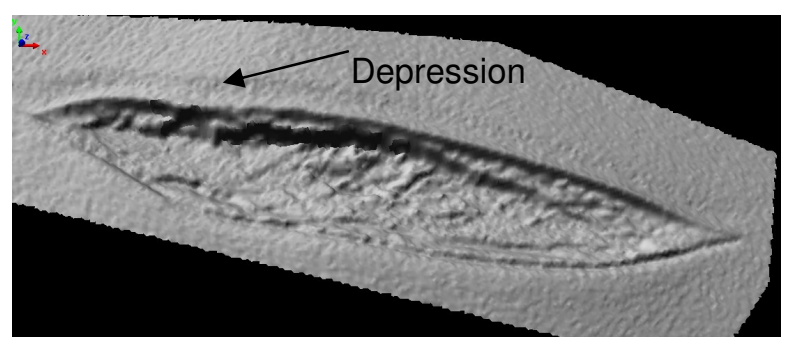

(a)

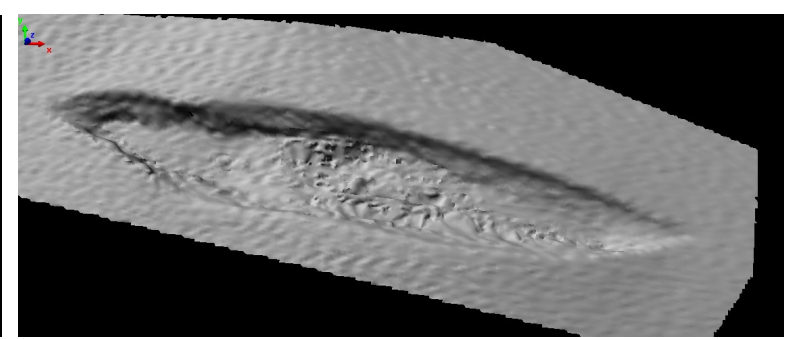

(b)

Fig. 11. Acquisition of the lesion. (a): Mesh obtained by using the Vivid 910 optical digitizer; (b): Mesh obtained by using the OPL-3D optical digitizer.

By looking at the two meshes, it is quite evident that the Vivid910 system provides more details of the lesion with respect to OPL-3D, in correspondence of both the borders and the interior tissues. We had the possibility of noticing this behavior directly on the point clouds, immediately after the acquisition. Obviously, our observation was made by comparing the point clouds to the lesion itself, by visually inspecting it. Forensic scientists confirmed our observations. To better observe the shapes obtained in the two cases, we overlapped them, and extracted profile sections at steps of $1 \mathrm{~mm}$ as shown in Fig. 12 . Green sections belong to the mesh from OPL-3D, gray sections are from the Vivid 910 system. It is well evident that the borders of the lesion are detected at a very different level of resolution. For example, in zone ' $A$ ', gray sections follow the light depression near the lesion (see also Fig. 11.a); in contrast they are not observable in green sections. The same behavior is observable on the right side of the lesion, even if it is less evident. Moreover, gray profiles show many details in correspondence with the region inside the lesion. In contrast, green profiles are smoothed.

To tell the truth, we were really frustrated by this result, since we thought that using incoherent light should led to better performance with respect to laser light. However, it should be noticed that we had 
to perform this test in very short time, and that it was impossible to optimize the system setup. This is quite evident looking at the fringe pattern in Fig. 10.b, where fringes are defocused, and their spatial period is rather large with respect to the FOV. This characteristics determined the smoothness of the reconstructed mesh.

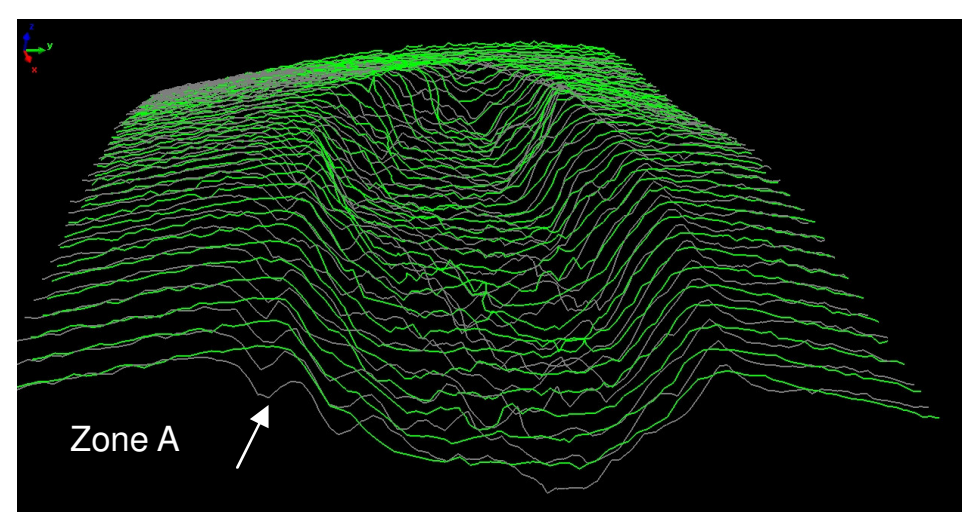

Fig. 12. Comparison of the two models. Green sections are from OPL-3D; gray sections are from the Vivid 910 system.

The quality of the mesh obtained by using the Vivid 910 proved to be very useful to accurately observe and quantitatively measure the features of the lesion. An example of the study is shown in Fig. 13. It presents a very close view of the lesion, as well as some measurements of the distance between pairs of points selected by the operator. The availability of such a representation was judged really novel by forensic scientists, due to (i) the adherence of the models to the original shapes, (ii) the possibility of analyzing them in subsequent times, and (iii) the quantitative, objective nature of the representation.

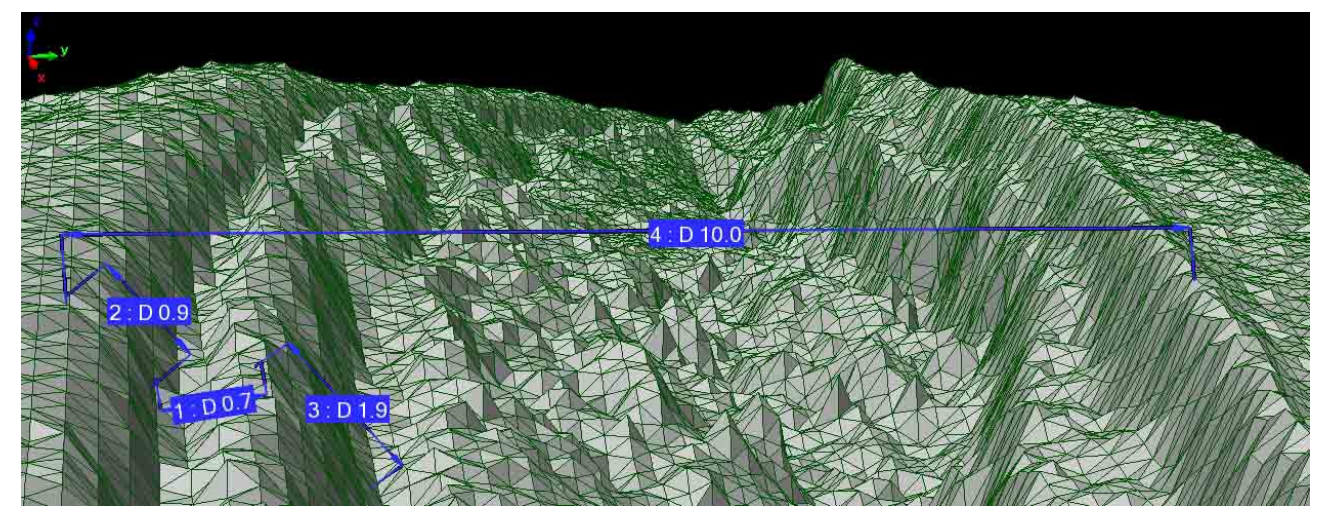

Fig. 13: Example of the measurements carried out on the mesh inFig. 11.a.

\section{The experience in maxillofacial prosthetics}

In maxillo-facial prosthetics, the use of optical, safe sensors combined with reverse engineering and rapid prototyping methods has gained increasing interest in the last years. Traditional reconstruction techniques are based on (i) use of impression making procedures, to obtain the negative patterns of the site of the deformity, (ii) plaster casting of negative patterns, to retrieve the positive defects, (iii) construction of wax positive replicas of the actual prosthesis, (iv) use of conventional flasking and investing procedures, to obtain the negative mould, and (v) casting of suitable materials into the negative mould, to obtain the prosthesis [11]. These procedures present a number of lacks. Firstly, impression making results in patient's discomfort and stress. The pressure that must be applied on the face to guarantee the required quality of the impression, inherently yields to the deformation of soft tissues, and to the impossibility of acquiring the original face features and look. Secondly, the quality of the wax positive replica is dependent on the artistic skills of an experienced anaplasthologist. The performance of the process strongly depends on both the shape and the extension of the defect. Human error contribution, subjectivity in the reconstruction, low reproducibility of the process, and poor initial shape information often lead to serious unfitting of the final prosthesis, under both functional and aesthetical points of view. Thirdly, the mould production process is cumbersome and time consuming. 
The overall process is not adaptive, i.e., whenever the existing prosthesis must be replaced, the overall process must be carried out from scratch.

Our activity has been developed together with the specialists of the Removable Prosthodontics School of Dentistry, University of Brescia. Over the years we studied the process of fabrication of the final prosthetic element for a nose, an eye and an ear $[18,19]$. The example that we present in this paper concerns the reconstruction of an ear. It shares with the previous cases the approach based on optical acquisition and modelling of the shapes. However, it is novel in the prototyping step, since the final prosthesis is directly obtained from the model by means of rapid prototyping. In contrast, in previous experiments, the final element was obtained by the traditional approach, by fabricating the wax positive replica and by casting the prosthesis material into the mould.

\subsection{The case of ear reconstruction}

The patient's defect is shown in Fig. 14. The left ear is seriously damaged in consequence of a burn. To fabricate the prosthetic element, the right ear, shown in Fig. 14.c was used as the template.

The test was performed as follows. Firstly, we acquired the right, safe ear. In the first approach, we configured the Vivid 910 in the TELE mode: the aim was to capture multiple small views, at the highest resolution. Unfortunately, due to the steep slope variations of the surface, and to their depth range, the system was often in the out-of-focus state, and the measurement could not be performed. Hence, we set it up in the MIDDLE configuration. Four views were acquired and aligned together. Then the triangle mesh was obtained and mirrored, in view of using it to model the prosthesis. The corresponding models are shown in Fig. 15.a, b and c.

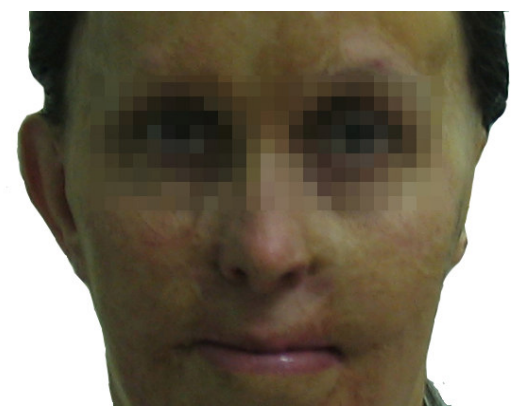

(a)

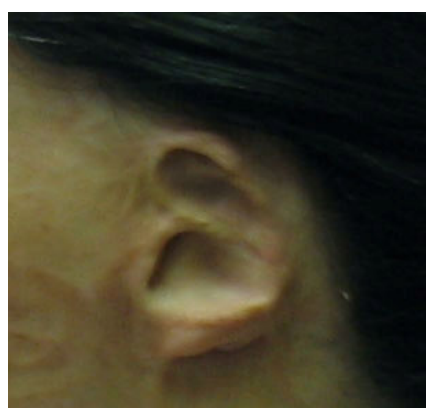

(b)

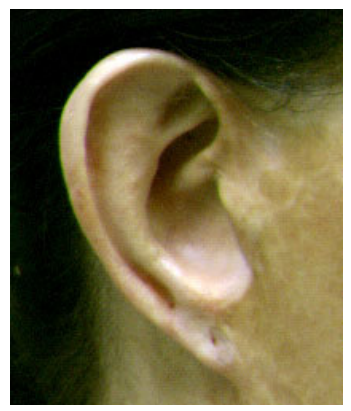

(c)

Fig. 14. The defect. (a): frontal view; (b): left side, (c): right, safe ear.

As a second step, the defect was gauged. The system configuration was the same as the one in the previous acquisition. Two views were sufficient to cover the whole surface. The mesh was created over the aligned views. The result is presented in Fig. 15.d.

The third step consisted in the acquisition of the whole patient face in three views, as shown in Fig. 16. This model was used as the skeleton to align the mesh in Fig. 15.d to the one in Fig. 15.c. The model of the defect was aligned to the skeleton. Then the model of the ear was interactively aligned until the aesthetical appearance on the whole face was judged optimal. At this point, the skeleton was discarded. The two models were edited to fill residual holes and to reconstruct missing surface parts (mainly due to undercuts). Finally, they were finely connected in correspondence with their borders. The result of this step is shown in Fig. 17.

The mesh has been topologically controlled to produce the physical copy. This has been fabricated by means of rapid prototyping technology. The Connex 500 3D Printing System (Objet-Geometries Inc.) has been used. This machine is capable of printing parts and assemblies made of multiple model materials all in a single build. The materials used to fabricate the ear prosthetic element are the TangoBlackPlus Shore A85 for the area corresponding to the auricle surface, and the TangoBlackPlus Shore A27 for the areas at the borders of the ear. The ear was obtained in about one hour; the process is very cheap (the cost is in the order of 70 Euros). Fig. 18 shows the front and the back side of the final prosthesis. 


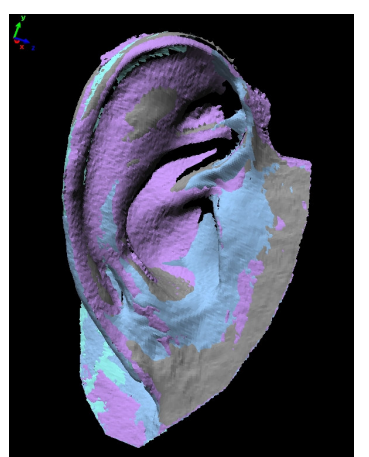

(a)

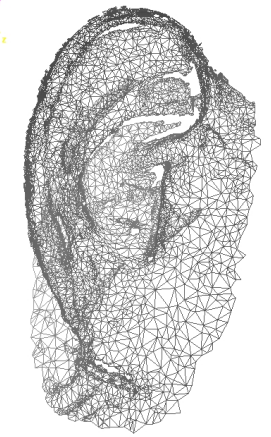

(b)

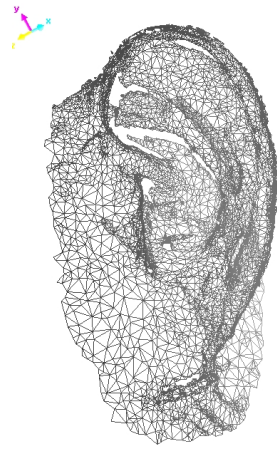

(c)

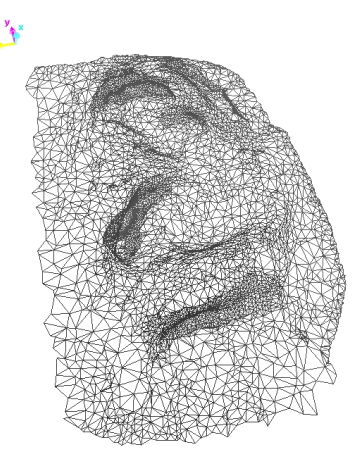

(d)

Fig. 15. Acquisition and creation of the model of the right ear. (a): alignment of the views; (b): mesh of the right ear; (c): mirrored ear; (d): mesh of the defect.

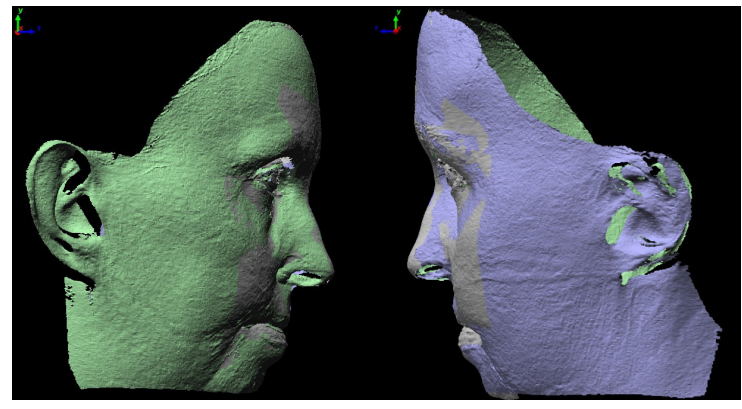

(a)

(b)

Fig. 16: Alignment of the views acquired in correspondence with the face. (a): right side. (b): left side.

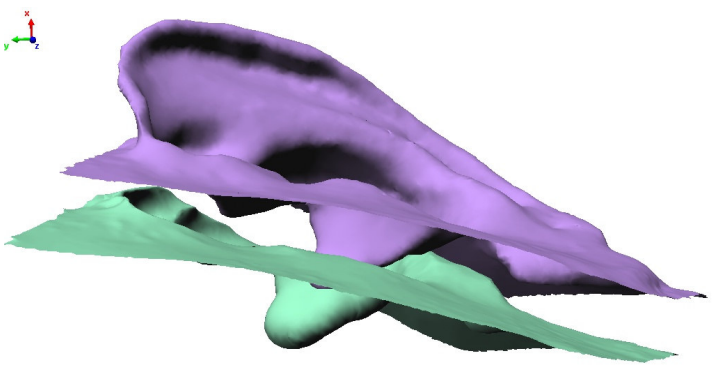

(a)

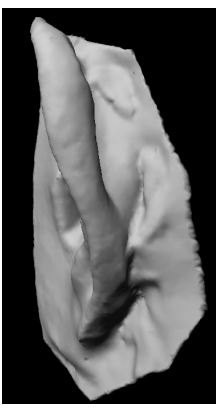

(b)

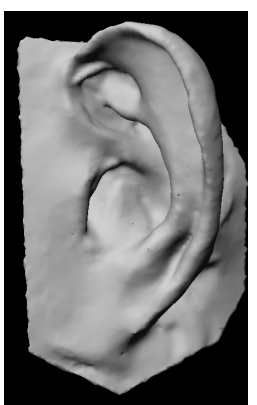

(c)

Fig. 17. Final model of the ear prosthesis. (a): alignment of the models; (b): back side of the final model; (c): front side of the final model.

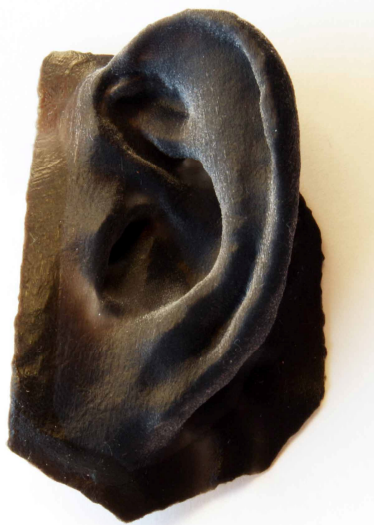

(a)

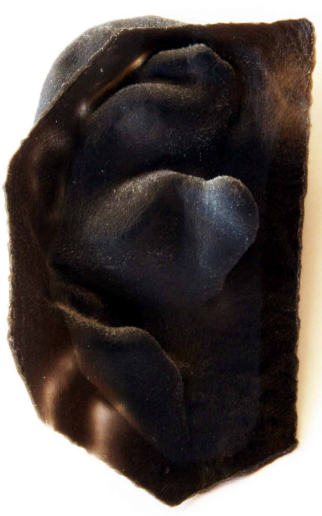

(b)

Fig. 18. The final prosthetic element. (a): front side; (b): back side. 
Fig. 19 shows the patient's face after the application of the prosthetic element. It is worth noting that, in this figure, the prosthesis color was not yet optimized. In fact, we wanted to check its functionality before optimizing it under the aesthetical point of view.

In this process, patient comfort was optimal, since the acquisition step was quick, contactless and safe. The prosthesis try-in was unnecessary. The prototyping step was very cheap, and the overall time required was about six hours, plus the machining of the prosthesis.

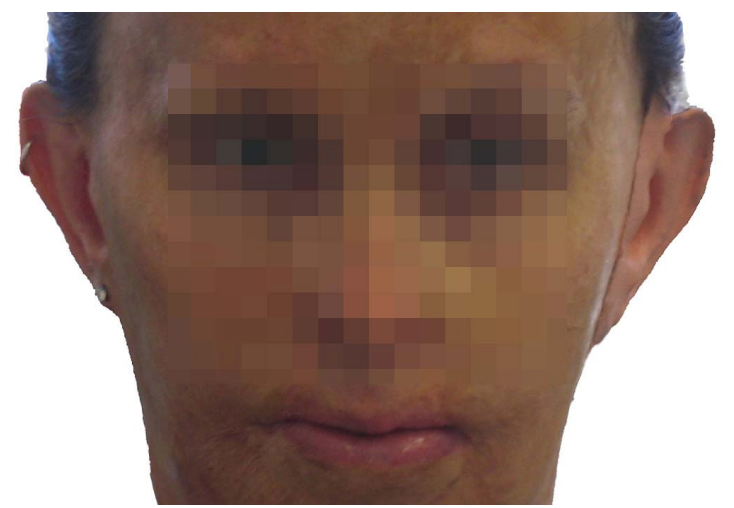

Fig. 19. Application of the prosthetic element to the patient's face.

\section{Conclusions}

In this paper the results of the activity concerning post mortem analysis of body segments and prosthetic reconstruction of the ear of a patient have been presented. The former activity has been very helpful to understand the capabilities of using optical scanning and reverse engineering in the analysis of skin lesions. The latter, which is still in progress, is very promising in view of allowing patients to overcome the social, psychological and economic problems deriving from their handicap.

\section{References}

1. Sansoni, G., Docchio, F., (2005): "In-field performance of an optical digitizer for the reverse engineering of free-form surfaces", Int. J Adv. Man. Tech., Vol.26, pp.1353-1361.

2. Sansoni, G., Docchio, F., (2004): "Three-dimensional optical measurements and reverse engineering for automotive applications", Robotics and Computer-Integrated manufacturing, Vol.20, pp.359-367.

3. Blais F., (2004): "A review of 20 years of range sensors development", J Electronic Imaging, Vol.13, No.11, pp.231-240.

4. Alunni-Perret, V., Muller-Bolla, M., Laugier, JP., Lupi-Pégurier, L., Bertrand, MF., Staccini, P., et al., (2005): "Scanning electron microscopy analysis of experimental bone hacking trauma", J Forensic Sci, Vol.50, No.4, pp.796-801.

5. Thali, M. J., Yen, K., Vock, P., Ozdobac, C., Kneubuehl, B. P., Sonnenschein, M., Dirnhofer, R., (2003): "Image-guided virtual autopsy findings of gunshot victims performed with multi-slice computed tomography (MSCT) magnetic resonance imaging (MRI) and subsequent correlation between radiology and autopsy findings", Forensic Science International, Vol.138, pp. 8-16.

6. Thali, M. J., Braun, M., Bruschweiler, W., Dirnhofer, R., (2000): "Matching tire tracks on the head using forensic photogrammetry", Forensic Science International, Vol.113, pp.281-287.

7. Bruschweiler, W., Braun, M., Dirnhofer, R., (2003): "Analysis of patterned injuries and injury-causing instruments with forensic 3D/CAD supported photogrammetry (FPHG): an instruction manual for the documentation process", Forensic Science International, Vol.132, pp.130-138.

8. Thali, M. J., Braun, M., Markwalder, T. H., Brueschweiler, W., Zollinger, U., Malika, N. J., Yena, K., Dirnhofer, R., (2003): "Bite mark documentation and analysis: the forensic 3D/CAD supported photogrammetry approach", Forensic Science International, Vol.135, pp.115-121.

9. Jiao, T., et al., (2004): "Design and Fabrication of Auricular Prostheses by CAD/CAM System", Int J Prosthodont, Vol.17, No.4, pp.460-463.

10. Verdonk, H. W. D., Pouken, J., Overveld, H.V. and Riediger, D., (2003): "Computer-assisted Maxillofacial prosthodontics: A new treatment protocol”, Int J Prosthodont, Vol.16, pp.326-328.

11. Taylor, T.D., (2000): “Clinical maxillofacial prosthetics”, Quintessence, pp.245-64. 
12. Tsuji, M., Noguchi, N., Ihara, K., Yamashita, Y., Shikimori, M., Goto, M., et al., (2004): "Fabrication of a maxillofacial prosthesis using a computer-aided design and manufacturing system": J Prosthodont, Vol.13, pp.179-83.

13. Pan, J., Zhao, Y., Su, F., (2007): "Application of phase measuring profilometry in reconstructing a 3D digitizing face model with open eyes", J US-China Medical Science, Vol.4, No.1, pp.51-54.

14. Reitemeier, B., Notni, G., Heinze, M., Schone, C., Schmidt, A., Fichtner, D., (2004): "Optical modeling of extraoral defects", J Prosthet Dent, Vol.91, pp.80-84.

15. Sansoni, G., Trebeschi, M. and Docchio, F., (2009): "State-of-The-Art and Applications of 3D Imaging Sensors in Industry, Cultural Heritage, Medicine, and Criminal Investigation", Sensors, Vol.9, No.1, pp.568-601.

16. Sansoni, G., Cattaneo, C., Trebeschi, M., Gibelli, D., Porta, D., Picozzi, M., (2009): "Feasibility of contactless 3D optical measurement for the analysis of bone and soft tissues lesions: new technologies and perspectives in forensic sciences", J Forensic Sci, Vol.54, No.4, pp.540-5.

17. Sansoni, G., Cattaneo, C., Trebeschi, M., Gibelli, D., Poppa, P., Porta, D., Maldarella, M., Picozzi, M., (2009): "Scene of crime analysis by a 3D optical digitizer: a useful perspective for forensic science", Am J Forensic Med Pathol, Accepted May 28, 2009.

18. Sansoni, G., Cavagnini, G., Docchio, F., Gastaldi, G., (2009): "Virtual and physical prototyping by means of a 3D optical digitizer: application to facial prosthetic reconstruction", Virtual and Physical Prototyping, Vol.4, pp.217-226.

19. Sansoni, G., Trebeschi, M., Cavagnini, G., Gastaldi, G., (2009): "3D Imaging acquisition, modelling and prototyping for facial defects reconstruction", Proceedings of SPIE Three-Dimensional Imaging Metrology 2009, San Jose CA, Vol.7239, 72390Y, pp.1-8.

20. Sansoni, G., Patrioli, A., Docchio, F., (2003): "OPL-3D: a novel, portable optical digitiser for fast acquisition of free-form surfaces", Rev. Scient. Instr., Vol. 74, No.4, pp.2593-2603. 